\title{
Erratum to: Physical Geology of Shallow Magmatic Systems
}

Christoph Breitkreuz and Sergio Rocchi

\section{Erratum to: \\ C. Breitkreuz and S. Rocchi (eds.), Physical Geology of Shallow Magmatic Systems, https://doi.org/10.1007/978-3-319-14084-1}

The original version of the book was inadvertently published with incorrect chapter DOIs and Copyright Year information for all chapters. They have been updated with original DOIs and Copyright Year as per online first chapters. Further, the series subtitle has been included in the series page. 